\title{
Three New Compounds from Piper montealegreanum Yuncker (Piperaceae)
}

\author{
Harley da S. Alves, Maria de F. V. de Souza and Maria C. de O. Chaves* \\ Laboratório de Tecnologia Farmacêutica, Universidade Federal da Paraíba, \\ CP 5009, 58051-970 João Pessoa-PB, Brazil
}

\begin{abstract}
Três novos compostos: dois flavonóides [(S)-8-formil-3',5-diidroxi-7-metoxi-6-metilflavanona (1) e 3'-formil-3,4',6'-triidroxi-2'-metoxi-5'-metilchalcona (2)] e um fenilpropanóide [3,4-metilenodioxi-5-metoxi-7,8-diidrocinamato de etila (3)] foram isolados dos ramos secos de Piper montealegreanum. As estruturas desses compostos foram estabelecidas através das técnicas espectroscópicas UV, IV, EM e RMN ( ${ }^{1} \mathrm{H}$ e ${ }^{13} \mathrm{C}, 1 \mathrm{D}$ e 2D), além da interpretação dos dados de $\mathrm{RMN}$ de ${ }^{1} \mathrm{H}$ e ${ }^{13} \mathrm{C}$ dos derivados metilados dos compostos $\mathbf{1}$ e $\mathbf{2}$.
\end{abstract}

Three new compounds: two flavonoids [(S)-8-formyl-3',5-dihydroxy-7-methoxy-6methylflavanone (1) and 3'-formyl-3,4',6'-trihydroxy-2'-methoxy-5'-methylchalcone (2)] and one phenylpropanoid [ethyl 3,4-methylenedioxy-5-methoxy-7,8-dihydrocinnamate (3)] were isolated from dried branches of Piper montealegreanum. Their structures were established by UV, IR, MS, $1 \mathrm{D}$ and $2 \mathrm{D}\left({ }^{1} \mathrm{H}\right.$ and $\left.{ }^{13} \mathrm{C}\right) \mathrm{NMR}$ spectroscopic techniques, besides interpretation of spectral data $\left({ }^{1} \mathrm{H}\right.$ and ${ }^{13} \mathrm{C}$ NMR) of methylated derivatives of $\mathbf{1}$ and $\mathbf{2}$ compounds.

Keywords: Piper montealegreanum, flavanone, chalcone, phenylalcanoid

\section{Introduction}

Piper montealegreanum Yuncker (Piperaceae) is a shrub, native to the north Brazil ${ }^{1}$ and has no previous chemical studies reported. A continuing search on the chemistry and bioactive agents from Brazilian north-northeast Piperaceae species have resulted in the isolation of amides, ${ }^{2-5}$ $\operatorname{aristolactams}^{6,7}$ and propenylphenols. ${ }^{8-12}$ In this paper, we report the isolation and structure elucidation of $(S)$ 8-formyl-3',5-dihydroxy-7-methoxy-6-methylflavanone (1), 3'-formyl-3,4',6' -trihydroxy-2' -methoxy-5' methylchalcone (2), and ethyl 3,4-methylenedioxy-5methoxy-7,8-dihydrocinnamate (3) from the branches of $P$. montealegreanum (Figure 1). The structures of the compounds were determined by interpretation of the spectral data analysis of UV, IR, MS, ${ }^{1} \mathrm{H}$ and ${ }^{13} \mathrm{C}$ NMR, including 2D NMR HMQC (heteronuclear multiple quantum coherence), HMBC (heteronuclear multiple bond correlation), and by comparison with those reported in the literature. $^{13}$

\footnotetext{
*e-mail: cchaves@1tf.ufpb.br
}

\section{Results and Discussion}

Compound 1 was obtained as orange-yellow crystals. The MS spectrum presented a molecular ion peak at $m / z$ 327.0887 (M-1) ${ }^{-}$, in LC-MS-IT-TOF apparatus (ion traptime of flight liquid chromatography mass spectrometry). The ${ }^{1} \mathrm{H}$ NMR spectrum showed the presence of four singlets at $\delta_{\mathrm{H}} 12.63(1 \mathrm{H}), 10.15(1 \mathrm{H}), 3.99(3 \mathrm{H})$ and 2.05 $(3 \mathrm{H})$ consistent with the presence of chelated hydroxyls, aldehyde, methoxyl and methyl groups, respectively. The presence of three signals at $\delta_{\mathrm{H}}, 5.43(\mathrm{dd}, 1 \mathrm{H}, J 10.8$ and $4.6 \mathrm{~Hz}$ ), 2.97 (dd, 1H, J 17.0 and $10.8 \mathrm{~Hz}$ ), 2.85 (dd,1H, $J$ 4.6 and $17.0 \mathrm{~Hz}$ ), and also signals for four coupled aromatic protons at $\delta_{\mathrm{H}}, 7.26(\mathrm{t}, 1 \mathrm{H}, J 8.0 \mathrm{~Hz}), 6.85(\mathrm{brd}, 1 \mathrm{H}, J 8.0$ $\mathrm{Hz})$ and $6.93(\mathrm{~m}, 2 \mathrm{H})$, suggested a flavanone nucleus ${ }^{14}$ with a 3'-monosubstituted B ring, deduced by analysis of the multiplicity and coupling constants of the aromatic protons. ${ }^{15}$ The above data and UV spectrum, with $\lambda_{\text {max }}$ at 266 $\mathrm{nm}$, reinforced a flavanone nature for compound $1 .{ }^{13}$ Since no further aromatic protons were evident, ring A should be fully substituted. The presence of the signal to methine carbon at $\delta_{\mathrm{C}} 193.8$ in the ${ }^{13} \mathrm{C}$ NMR APT (attached proton test) spectrum was taken as a proof for the presence of an aldehyde group, and the low-field hydroxyl hydrogen $\left[\delta_{\mathrm{H}}\right.$ 
<smiles>[Y17][R]([H])([H])[H]</smiles>

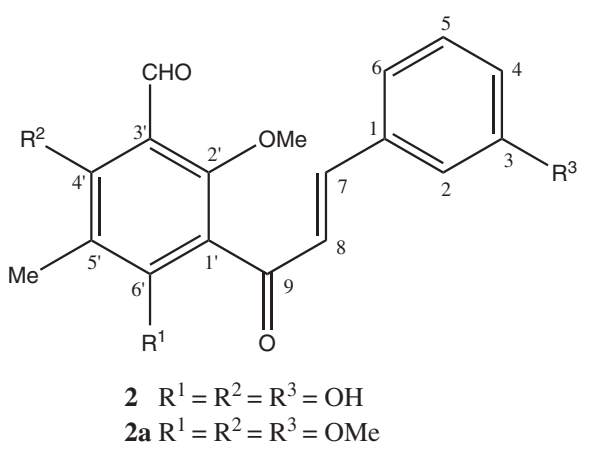<smiles>CCOC(=O)CCc1cc(OC)c2c(c1)OCO2</smiles>

Figure 1. Structures of the isolated compounds 1-3 from Piper montealegreanum.

$12.63(\mathrm{~s}, 1 \mathrm{H})]$ evidenced the chelated hydroxyl at $\mathrm{C}-5$ with the $\mathrm{C}=\mathrm{O}$ of the $\alpha, \beta$-unsaturated carbonyl group. ${ }^{13}$

In the HMBC spectrum, the presence of cross peaks at $\delta_{\mathrm{H}} 12.63$ with $\delta_{\mathrm{C}} 166.3$ and 109.3 , besides the cross peak at $\delta_{\mathrm{H}} 2.05$ with $\delta_{\mathrm{C}} 166.3,166.1$ and 109.3, evidenced the existence of a methyl group at C-6 and also suggested the attachment position of the methoxyl group at C-7. This was confirmed by correlation of the peak at $\delta_{\mathrm{H}} 10.15$ (aldehyde hydrogen) and $\delta_{\mathrm{H}} 3.99$ (methoxyl hydrogen) with $\delta_{\mathrm{C}} 166.1$, and consequently, the placement of the formyl group at C-8 (Figure 2).

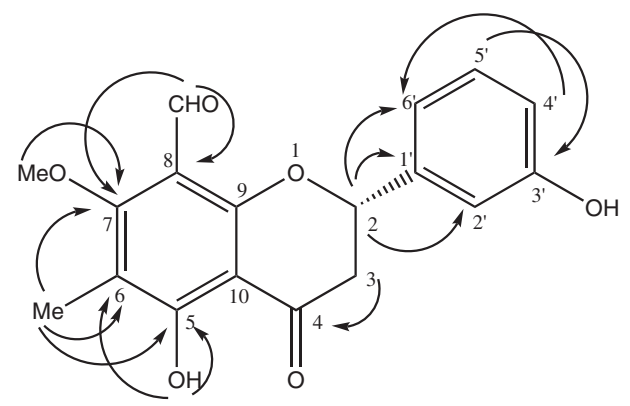

Figure 2. HMBC correlations of $\mathbf{1}$.

The compound 1a (Figure 1), a methylated derivative of $\mathbf{1}$, showed correlations in HMQC spectrum between $\delta_{\mathrm{H}}$ $3.95 / \delta_{C} 64.3$ and $\delta_{\mathrm{H}} 3.82 / \delta_{\mathrm{C}} 55.3$. The HMBC spectrum displayed correlations between $\delta_{\mathrm{H}} 3.95$ and $2.14 / \delta_{\mathrm{C}} 164.6$ confirming the methoxyl group at C-5. The presence of cross peaks between signals in $\delta_{\mathrm{H}} 3.82 / \delta_{\mathrm{C}} 159.9$ supported the methoxyl group at the $\mathrm{C}-3^{\prime}$.
Compound 2 was obtained as yellow crystals. The MS spectrum gave a molecular ion peak at $m / z 327.0870$ (M-1)', in LC-MS-IT-TOF apparatus. The ${ }^{1} \mathrm{H}$ NMR spectrum indicated signals at $\delta_{\mathrm{H}} 7.78$ and 7.84 suggesting the presence of protons on a $\alpha, \beta$-unsaturated ketone moiety. ${ }^{13,16}$ The above data and UV absorption bands $\left(\lambda_{\text {max }}\right)$ at 317 and $282 \mathrm{~nm}$ suggested a chalcone structure for compound $2 .{ }^{13}$ Signals for four coupled aromatic protons: a triplet at $\delta_{\mathrm{H}}$ $7.33(1 \mathrm{H}, \mathrm{J} 7.8 \mathrm{~Hz})$, a multiplet at $\delta_{\mathrm{H}} 7.28-7.23(\mathrm{~m}, 2 \mathrm{H})$ and a double double doublet at $\delta_{\mathrm{H}} 6.97(1 \mathrm{H}, J 7.8,2.0$ and $1.6 \mathrm{~Hz}$ ) suggested a 3'-substituted B ring. ${ }^{15}$ Additionally, the ${ }^{1} \mathrm{H}$ NMR spectrum showed tree singlets at $\delta_{\mathrm{H}}, 2.01(3 \mathrm{H})$, $4.01(3 \mathrm{H})$ and $10.17(1 \mathrm{H})$, consistent with the presence of methyl, methoxyl and aldehyde groups, respectively, besides two hydroxyl group singlets at $\delta_{\mathrm{H}} 12.83$ and 14.21. The two low-field hydroxyl protons evidenced a formyl group at C-3'. This conclusion is achieved since the down-field shift of OH-4' $\left(\delta_{\mathrm{H}} 12.83\right)$ can be explained by hydrogen-bonding with the oxygen atom of the formyl substituent at a neighboring carbon atom and at the same time that the down-field shift of OH-6' $\left(\delta_{\mathrm{H}} 14.21\right)$ is caused by chelation between the 6 '-hydroxyl proton and the carbonyl oxygen of the $\alpha, \beta$-unsaturated carbonyl group function. ${ }^{13}$ This intramolecular hydrogen bonding corroborated the assignments of the signals at $\delta 7.78(\mathrm{~d}, 1 \mathrm{H})$ and $\delta 7.84(\mathrm{~d}, 1 \mathrm{H})$ to $\alpha$ and $\beta$ positions, respectively. ${ }^{16}$ Spectral analysis of $\mathbf{2 a}$ showed $\mathrm{H}_{\alpha}$ and $\mathrm{H}_{\beta}$ signals with a marked difference in chemical shifts $[\delta 7.29(\mathrm{~d}, 1 \mathrm{H}$, $J 16.0 \mathrm{~Hz}, \mathrm{H}-7)$ and $\delta 6.98(\mathrm{~d}, 1 \mathrm{H}, J 16.0 \mathrm{~Hz}, \mathrm{H}-8)]$ related with the absence of the intramolecular hydrogen bonding with the $\mathrm{C}=\mathrm{O}$ of the $\alpha, \beta$-unsaturated carbonyl group. The 
coupling constant $(16.0 \mathrm{~Hz})$ observed in 2a indicated the $E$-isomer for the double bond. ${ }^{16}$ The placement of the other groups in the A-ring was made on the basis of the HMBC correlations (Figure 3): $\delta_{\mathrm{H}} 14.21\left(\mathrm{~s}, 1 \mathrm{H}, \mathrm{OH}-6\right.$ ') $/ \delta_{\mathrm{C}} 169.8$ and 109.0; $\delta_{\mathrm{H}} 12.83\left(\mathrm{~s}, 1 \mathrm{H}, \mathrm{OH}-4{ }^{\prime}\right) / \delta_{\mathrm{C}} 166.6,109.2$ and $109.5 ; \delta_{\mathrm{H}} 2.01(\mathrm{~s}, 3 \mathrm{H}) / \delta_{C} 109.2 ; \delta_{\mathrm{H}} 10.17(\mathrm{~s}, 1 \mathrm{H}) / \delta_{\mathrm{C}}$ 166.6 and $\delta_{\mathrm{C}} 109.5 ; \delta_{\mathrm{H}} 4.01 / \delta_{\mathrm{C}} 168.5$ that evidenced the hydroxyl, formyl, methyl and methoxyl groups at C-6' -4 ', C-3', C-5' and C-2', respectively.

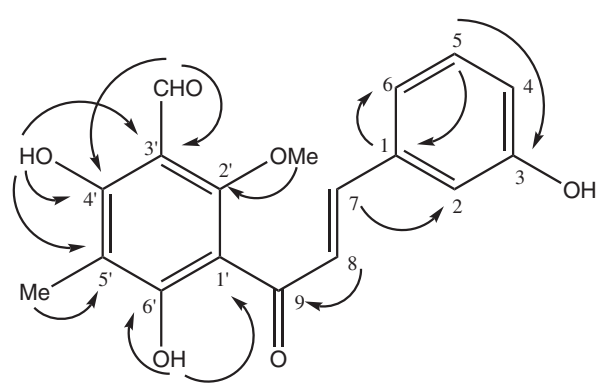

Figure 3. HMBC correlations of 2.

The hydroxyl group in the C-3 was confirmed for the methylated derivative of $\mathbf{2}$ (Figure 1) which showed correlation between $\delta_{\mathrm{H}} 3.80 / \delta_{\mathrm{C}} 55.3$ in the HMQC spectrum and $\delta_{\mathrm{H}} 3.80 / \delta_{\mathrm{C}} 159.9(\mathrm{C}-3)$ in the HMBC spectrum. Other correlations observed in the HMQC spectrum of $2 \mathrm{a}$ were $\delta_{\mathrm{H}} 3.86 / \delta_{\mathrm{C}} 62.7$ and $\delta_{\mathrm{H}} 3.75 / \delta_{\mathrm{C}} 61.9$. In the HMBC spectrum, the signals at $\delta_{\mathrm{H}} 3.86$ and 3.75 showed correlations with $\delta_{C} 163.0$ and 161.8 , respectively, providing support for the presence of the methoxyl groups at the C-6' and C-4' position.

Formyl flavonoids have been reported from a few species in the plant kingdom. Early reports included a description of 2',4-dihydroxy-4' -methoxy-5' formylchalcone from Psoralea corylifolia (Fabaceae). ${ }^{17}$ Its isomeric compound, with methoxy group at the 2 '-position, has been reported from the same species. ${ }^{18}$ 2', 4',6' - trihydroxy-3' '-formylchalcone has been reported from Psidium acutangulum (Myrtaceae) ${ }^{19}$ and its retrochalcone derivative was obtained from Anredera scandens (Basellaceae). ${ }^{20}$ 3'-formyl-4',6'-dihydroxy2'-methoxy-5'-methylchalcone and (2S)-8-formyl-5hydroxy-7-methoxy-6-methylflavanone were isolated from Cleistocalyx operculatus (Myrtaceae). ${ }^{13}$

The compound $\mathbf{3}$ was obtained as yellow powder. The ${ }^{1} \mathrm{H}$ spectrum showed two doublets in $\delta_{\mathrm{H}} 6.37(J 1.4 \mathrm{~Hz}$, $1 \mathrm{H})$ and $\delta_{\mathrm{H}} 6.34(\mathrm{~J} 1.4 \mathrm{~Hz}, 1 \mathrm{H})$ typical of meta aromatics hydrogens in addition to two singlets in $\delta_{\mathrm{H}} 5.91(2 \mathrm{H})$ and $3.86(3 \mathrm{H})$ typical of methylenedioxy and methoxyl groups, respectively. These data suggested the presence of tetrasubstituted aromatic ring. The signals in $\delta_{\mathrm{H}} 4.11$ $(\mathrm{q}, 2 \mathrm{H}, J 7.0 \mathrm{~Hz}$ ) and $1.23(\mathrm{t}, 3 \mathrm{H}, J 7.0 \mathrm{~Hz})$ supported the presence of ethyl group attached to heteroatom and also two signals at $\delta_{\mathrm{H}} 2.84(\mathrm{t}, 2 \mathrm{H}, J 7.5 \mathrm{~Hz})$ and $2.55(\mathrm{t}, 2 \mathrm{H}$, $J 7.5 \mathrm{~Hz}$ ) typical of methylene hydrogens.

The ${ }^{13} \mathrm{C}$ NMR spectrum of $\mathbf{3}$ showed 13 signals. The signals at $\delta_{\mathrm{H}} 2.84,2.55$ and 4.11 are, in the HMBC spectrum, correlated with a signal characteristic of carbonyl ester at $\delta_{\mathrm{C}} 172.8$, confirming the assignment of the chemical shifts for H-7, H-8 and H-10, respectively, and $\delta_{\mathrm{C}} 172.8$ for C-9 of a dihydrocinnamoyl group. The HMBC spectrum also showed correlations between $\delta_{\mathrm{H}}$ $3.86 / \delta_{\mathrm{C}} 148.8, \delta_{\mathrm{H}} 5.91 / \delta_{\mathrm{C}} 143.5$ and 133.5 , confirming the methoxyl and methylenedioxy groups at $\mathrm{C}-5$ and C-3-4, respectively.

\section{Experimental}

\section{General procedures}

Melting points (mp) were determined on a MQAPF-302 melting point digital apparatus. UV spectra were recorded on a Vankel-50 UV-Vis spectrophotometer. IR spectra were obtained on an FT-IR-1750 spectrophotometer, PerkinElmer apparatus. The spectra mass were obtained on a SHIMADZU LCMS-IT-TOF (225-07100-34) equipped with a Z-spray ESI (electrospray) source and operated in negative mode.

${ }^{1} \mathrm{H}$ and ${ }^{13} \mathrm{C}$ NMR (1D and 2D) spectra were recorded on a Varian Mercury 200 spectrometer in $\mathrm{CDCl}_{3}$ and $\left(\mathrm{CD}_{3}\right)_{2} \mathrm{CO}$, with TMS as internal standard. Sephadex LH-20 and silica gel $60\left(\mathrm{PF}_{254}\right.$ art. 7749 and art. 7731) were purchased from Merck.

The methylated flavonoids were obtained by treatment of the sample, dissolved in dry propanone, with 1.1 equiv. of dimethyl sulphate $\left(\mathrm{Me}_{2} \mathrm{SO}_{4}\right)$ and 1.1 equiv. of potassium carbonate $\left(\mathrm{K}_{2} \mathrm{CO}_{3}\right)$ to each free hydroxyl. The reactions were carried at room temperature during $12 \mathrm{~h}$. After removal of the solvent in vacuum, the residue was suspended in $\mathrm{H}_{2} \mathrm{O}(50 \mathrm{~mL})$, treated with $5 \mathrm{~mL}$ of ammonia and extracted with $\mathrm{CHCl}_{3}(3 \times 15 \mathrm{~mL})$. The $\mathrm{CHCl}_{3}$ solution was dried with $\mathrm{Na}_{2} \mathrm{SO}_{4}$, filtrated and concentrated to dryness. ${ }^{13}$

\section{Plant material}

Branches of Piper montealegreanum Yuncker was collected in Belém (Pará State, Brazil), in December 2002 and identified in the Botanical Garden, Rio de Janeiro (Rio de Janeiro State, Brazil). A voucher specimen (MSP-010) was deposited at Emilio Goeldi Museum, Belém. 


\section{Extraction and isolation}

The powered material of $P$. montealegreanum $(1.3 \mathrm{~kg})$ was exhaustively extracted with $\mathrm{EtOH}(4 \times 2.0 \mathrm{~L})$, the solvent removed under reducted pressure furnished a green residue $(115.0 \mathrm{~g})$. The crude extract amount of $13.5 \mathrm{~g}$ was chromatographed over Sephadex gel LH-20 and eluted with methanol (column 1) yielding 43 fractions. Fraction 17 was further fractionated over Sephadex gel LH-20 column providing 5 fractions. Fraction 3 after submitted to recristalization with a chloroform and methanol mixture yielding $(S)$-8-formyl-3',5-dihydroxy-7-methoxy-6methyl-flavanone (1) (30 mg).

Fraction 18 (column 1) was chromatographed over Sephadex LH-20 and yielded five fractions. Fraction 4 gave 3'-formyl-3,4',6'-trihydroxy-2'-methoxy5'-methylchalcone (2) (40 mg), after submitted to recristalization with a chloroform and methanol mixture.

Fractions 8-11 (column 1) were also fractionated over Sephadex gel yielding 29 fractions. Fraction 8-14, after recrystallization with chloroform, gave ethyl 3,4-methylenedioxy-5-methoxy-7,8-dihydrocinnamate (3) (12 mg).

Compound 1. Orange yellow crystals $\left(\mathrm{CDCl}_{3}: \mathrm{MeOH}\right)$; $\mathrm{mp} 162{ }^{\circ} \mathrm{C} ;[\alpha]_{\mathrm{D}}{ }^{25}-20(\mathrm{MeOH}, 0.025) ; \mathrm{UV} \lambda_{\max } / \mathrm{nm}$ (MeOH): 266; UV $\lambda_{\text {max }} / \mathrm{nm}\left(\mathrm{AlCl}_{3}\right): 286,323$ (sh); IR $v_{\text {max }} / \mathrm{cm}^{-1}(\mathrm{KBr}): 3425,1688,1620,1570,1464$; MS [M-1] ${ }^{-}$ 327.0887; ${ }^{1} \mathrm{H}$ and ${ }^{13} \mathrm{C}$ NMR spectral data, see Table 1.

Compound 2. Yellow crystals $\left(\mathrm{CHCl}_{3}: \mathrm{MeOH}\right) ; \mathrm{mp}$ $164{ }^{\circ} \mathrm{C}$; UV $\lambda_{\text {max }} / \mathrm{nm}(\mathrm{MeOH}): 317,282 ; \mathrm{UV} \lambda_{\max } / \mathrm{nm}$ $\left(\mathrm{AlCl}_{3}\right): 349,306$; IR (KBr) $v_{\max } / \mathrm{cm}^{-1}: 3445,1616,1581$, 1422; MS [M-1] $327.0870 ;{ }^{1} \mathrm{H}$ and ${ }^{13} \mathrm{C}$ NMR spectral data, see Table 1.

Compound $1 a$ and $2 a \cdot{ }^{1} \mathrm{H}$ and ${ }^{13} \mathrm{C}$ NMR spectral data, see Table 2 .

Compound 3. Amorphous green powder; IR (KBr) $v_{\text {max }} / \mathrm{cm}^{-1}: 2924,2853,1734 ; \mathrm{MS}\left[\mathrm{M}^{+}\right] 252 ;{ }^{1} \mathrm{H}$ and ${ }^{13} \mathrm{C} \mathrm{NMR}$ spectral data, see Table 3 .

Table 1. ${ }^{1} \mathrm{H}$ and ${ }^{13} \mathrm{C}$ NMR data for compounds $\mathbf{1}$ and $\mathbf{2}$

\begin{tabular}{|c|c|c|c|c|}
\hline \multirow[t]{2}{*}{ Position } & \multicolumn{2}{|c|}{ Chemical shift, $\delta\left({ }^{1} \mathrm{H}\right)$} & \multicolumn{2}{|c|}{ Chemical shift, $\delta\left({ }^{13} \mathrm{C}\right)$} \\
\hline & 1 & 2 & 1 & 2 \\
\hline 1 & - & - & - & 137.1 \\
\hline 2 & $5.43(\mathrm{dd}, 1 \mathrm{H}, J 10.8$ and $4.6 \mathrm{~Hz})$ & $7.28-7.23(\mathrm{~m}, 1 \mathrm{H})$ & 78.3 & 115.5 \\
\hline 3 & $2.97 \mathrm{ax},(\mathrm{dd}, 1 \mathrm{H}, J 17.0$ and $10.8 \mathrm{~Hz})$ & - & 45.0 & 158.9 \\
\hline & $2.85 \mathrm{eq},(\mathrm{dd}, 1 \mathrm{H}, J 4.6$ and $17.0 \mathrm{~Hz})$ & & & \\
\hline 4 & - & $6.97(\mathrm{ddd}, 1 \mathrm{H}, J$ 7.8. 2.0 and $1.6 \mathrm{~Hz})$ & 188.9 & 119.1 \\
\hline 5 & - & $7.33(\mathrm{t}, 1 \mathrm{H}, J 7.8 \mathrm{~Hz})$ & 166.3 & 131.1 \\
\hline 6 & - & 7.28-7.23 (m, 1H) & 109.3 & 121.3 \\
\hline 7 & - & $7.78(\mathrm{~d}, 1 \mathrm{H}, J 15.5 \mathrm{~Hz})$ & 166.1 & 145.8 \\
\hline 8 & - & $7.84(\mathrm{~d}, 1 \mathrm{H}, J 15.5 \mathrm{~Hz})$ & 109.9 & 126.3 \\
\hline 9 & & - & 165.6 & 193.8 \\
\hline 10 & - & - & 107.5 & - \\
\hline 1 ' & - & - & 139.7 & 109.0 \\
\hline $2^{\prime}$ & $6.93(\mathrm{~m}, 2 \mathrm{H})$ & - & 112.8 & 168.5 \\
\hline 3 & - & - & 156.3 & 109.5 \\
\hline $4^{\prime}$ & $6.85(\mathrm{dl}, 1 \mathrm{H}, J 8.0 \mathrm{~Hz})$ & - & 115.8 & 166.6 \\
\hline 5 & $7.26(\mathrm{t}, 1 \mathrm{H}, J 8.0 \mathrm{~Hz})$ & - & 130.2 & 109.2 \\
\hline $6{ }^{\prime}$ & $6.93(\mathrm{~m}, 2 \mathrm{H})$ & - & 117.8 & 169.8 \\
\hline $\mathrm{OMe}$ & $3.99(\mathrm{~s}, 3 \mathrm{H})$ & $4.01(\mathrm{~s}, 3 \mathrm{H})$ & 64.6 & 67.0 \\
\hline $\mathrm{Me}$ & $2.05(\mathrm{~s}, 3 \mathrm{H})$ & $2.01(\mathrm{~s}, 3 \mathrm{H})$ & 7.2 & 6.7 \\
\hline $\mathrm{CHO}$ & $10.15(\mathrm{~s}, 1 \mathrm{H})$ & $10.17(\mathrm{~s}, 1 \mathrm{H})$ & 193.8 & 194.3 \\
\hline $\mathrm{OH}-5$ & $12.63(\mathrm{~s}, 1 \mathrm{H})$ & - & - & - \\
\hline $\mathrm{OH}-4$ & - & $12.83(\mathrm{~s}, 1 \mathrm{H})$ & - & - \\
\hline OH-6' & - & $14.21(\mathrm{~s}, 1 \mathrm{H})$ & - & - \\
\hline
\end{tabular}


Table 2. ${ }^{1} \mathrm{H}$ and ${ }^{13} \mathrm{C}$ NMR data for methylated compounds $\mathbf{1 a}$ and $\mathbf{2 a}$

\begin{tabular}{|c|c|c|c|c|}
\hline \multirow[t]{2}{*}{ Position } & \multicolumn{2}{|c|}{ Chemical shift, $\delta\left({ }^{1} \mathrm{H}\right)$} & \multicolumn{2}{|c|}{ Chemical shift, $\delta\left({ }^{13} \mathrm{C}\right)$} \\
\hline & 1a & $2 a$ & 1a & $2 a$ \\
\hline 1 & - & - & - & 135.5 \\
\hline 2 & $5.48(\mathrm{dd}, 1 \mathrm{H}, J 12.0$ and $4.0 \mathrm{~Hz})$ & $7.02(\mathrm{~m}, 1 \mathrm{H})$ & 78.9 & 113.2 \\
\hline 3 & $\begin{array}{c}3.02 \mathrm{ax}(\mathrm{dd}, 1 \mathrm{H}, J 16.7 \text { and } 12.0 \mathrm{~Hz}) \text {; } \\
2.87 \mathrm{eq}(\mathrm{dd}, 1 \mathrm{H}, J 16.7 \text { and } 4.0 \mathrm{~Hz})\end{array}$ & - & 45.3 & 159.9 \\
\hline 4 & - & $6.91(\mathrm{~m}, 1 \mathrm{H})$ & 188.8 & 116.9 \\
\hline 5 & - & $7.27(\mathrm{t}, 1 \mathrm{H}, J 7.8 \mathrm{~Hz})$ & 164.6 & 129.9 \\
\hline 6 & - & $7.12(\mathrm{~m}, 1 \mathrm{H})$ & 117.3 & 121.4 \\
\hline 7 & - & $7.29(\mathrm{~d}, 1 \mathrm{H}, J 16.0 \mathrm{~Hz})$ & 164.8 & 146.4 \\
\hline 8 & - & $6.98(\mathrm{~d}, 1 \mathrm{H}, J 16.0 \mathrm{~Hz})$ & 117.9 & 128.3 \\
\hline 9 & - & - & 165.6 & 193.7 \\
\hline 10 & - & - & 115.6 & - \\
\hline 1 ' & - & - & 139.6 & 122.1 \\
\hline $2^{\prime}$ & $7.03-6.99(\mathrm{~m}, 2 \mathrm{H})$ & - & 111.8 & 158.9 \\
\hline $3^{\prime}$ & - & - & 159.9 & 119.23 \\
\hline $4^{\prime}$ & $6.94-6.88(\mathrm{~m}, 1 \mathrm{H})$ & - & 113.9 & 161.8 \\
\hline 5 & $7.35(\mathrm{t}, 1 \mathrm{H}, J 8.2 \mathrm{~Hz})$ & - & 130.0 & 125.1 \\
\hline 6 & $7.03-6.99(\mathrm{~m}, 2 \mathrm{H})$ & - & 118.0 & 163.0 \\
\hline OMe-3' & $3.82(\mathrm{~s}, 3 \mathrm{H})$ & - & 55.3 & - \\
\hline OMe-5 & $3.95(\mathrm{~s}, 3 \mathrm{H})$ & - & 64.3 & - \\
\hline $\mathrm{OMe}-7$ & $3.83(\mathrm{~s}, 3 \mathrm{H})$ & - & 62.3 & - \\
\hline $\mathrm{Me}$ & $2.14(\mathrm{~s}, 3 \mathrm{H})$ & $2.19(\mathrm{~s}, 3 \mathrm{H})$ & 8.4 & 8.9 \\
\hline $\mathrm{CHO}$ & $10.33(\mathrm{~s}, 1 \mathrm{H})$ & $10.30(\mathrm{~s}, 1 \mathrm{H})$ & 188.2 & 188.2 \\
\hline OMe-2' & - & $3.78(\mathrm{~s}, 3 \mathrm{H})$ & - & 64.5 \\
\hline OMe-4' & - & $3.75(\mathrm{~s}, 3 \mathrm{H})$ & - & 61.9 \\
\hline OMe-6' & - & $3.86(\mathrm{~s}, 3 \mathrm{H})$ & - & 62.7 \\
\hline OMe-3 & - & $3.80(\mathrm{~s}, 3 \mathrm{H})$ & - & 55.3 \\
\hline
\end{tabular}

Table 3. ${ }^{1} \mathrm{H}(200 \mathrm{MHz})$ and ${ }^{13} \mathrm{C}(50 \mathrm{MHz})$ NMR data for compound 3 and correlations obtained in $\mathrm{HMQC}$ and $\mathrm{HMBC}$ experiments, $J(\mathrm{~Hz})$ in parenthesis

\begin{tabular}{|c|c|c|c|c|}
\hline \multirow[t]{2}{*}{ Positions } & \multicolumn{3}{|c|}{ HMQC } & \multirow{2}{*}{$\begin{array}{c}\text { HMBC } \\
\delta_{\mathrm{C}} \times \delta_{\mathrm{H}}\left({ }^{3} J\right) \\
\end{array}$} \\
\hline & $\delta_{\mathrm{H}}$ & $\delta_{\mathrm{C}} \times \delta_{\mathrm{H}}\left({ }^{1} J\right)$ & $\delta_{\mathrm{C}} \times \delta_{\mathrm{H}}\left({ }^{2} J\right)$ & \\
\hline 1 & - & 135.2 & & \\
\hline 2 & $6.37(\mathrm{~d}, 1 \mathrm{H}, J 1.4 \mathrm{~Hz})$ & 102.3 & 148.8 & 107.5 \\
\hline 3 & - & 148.8 & & \\
\hline 4 & - & 133.5 & & \\
\hline 5 & - & 143.5 & & \\
\hline 6 & $6.34(\mathrm{~d}, 1 \mathrm{H}, J 1.4 \mathrm{~Hz})$ & 107.5 & 143.5 & $133.5 ; 102.3 ; 31.1$ \\
\hline 7 & $2.84(\mathrm{t}, 2 \mathrm{H}, J 7.5 \mathrm{~Hz})$ & 31.1 & $135.2 ; 36.3$ & $172.8 ; 107.5 ; 102.3$ \\
\hline 8 & $2.55(\mathrm{t}, 2 \mathrm{H}, J 7.5 \mathrm{~Hz})$ & 36.3 & $172.8 ; 31.1$ & 135.2 \\
\hline 9 & - & 172.8 & & \\
\hline 10 & $4.11(\mathrm{q}, J 7.0 \mathrm{~Hz})$ & 60.5 & 14.2 & 172.2 \\
\hline 11 & $1.23(\mathrm{t}, 3 \mathrm{H}, J 7.0 \mathrm{~Hz})$ & 14.2 & & \\
\hline 12 & $5.91(\mathrm{~s}, 2 \mathrm{H})$ & 101.2 & & $148.8 ; 133.5$ \\
\hline OMe-5 & $3.86(\mathrm{~s}, 3 \mathrm{H})$ & 56.5 & & 143.5 \\
\hline
\end{tabular}




\section{Acknowledgements}

The authors thank CNPq (Conselho Nacional de Desenvolvimento Científico e Tecnológico) for financial support, Laboratório de Espectrometria de Massas do Nordeste (LEMANOR) for MS spectra and Maria das Graças Bichara Zoghbi for plant material.

\section{Supplementary Information}

Supplementary information $\left({ }^{1} \mathrm{H}\right.$ and ${ }^{13} \mathrm{C}$ NMR data for compounds 1, 2, 3, 1a and 2a, Figures S1-S15) is available free of charge at http://jbcs.org.br as a PDF file.

\section{References}

1. Yuncker, T. G.; Hoehnea 1972, 2, 36.

2. Araújo-Jr, J. X.; Chaves, M. C. O.; Da Cunha, E. V. L.; Gray, A. I.; Phytochemistry 1997, 44, 559.

3. Chaves, M. C. O.; Santos, B. V. O.; Biochem. Syst. Ecol. 1998, 27, 113 .

4. Chaves, M. C. O.; Da Cunha, E. V. L.; Fitoterapia 2001, 72, 197.

5. Chaves, M. C. O.; Figueiredo-Jr, A. G.; Santos, B. V. O.; Fitoterapia 2003, 74, 181.

6. Araújo-Jr, J. X.; Chaves, M. C. O.; Da Cunha, E. V. L.; Gray, A. I.; Biochem. Syst. Ecol. 1999, 27, 325.

7. Cardozo-Júnior, E. L.; Chaves, M. C. O.; Pharm. Biol. 2003, $41,216$.

8. Oliveira, L. C. P.; Mause, R.; Nunomura, S. M.; J. Braz. Chem. Soc. 2005, 16, 1439.
9. Chaves, M. C. O.; Santos, B. V. O.; Biochem. Syst. Ecol. 1999, 27, 539.

10. Chaves, M. C. O.; Santos, B. V. O.; Fitoterapia 2002, 73, 547.

11. Santos, B. V. O.; Chaves, M. C. O.; da Cunha, E. V. L.; Gray, A.I.; Biochem. Syst. Ecol. 1997, 25, 471.

12. Santos, B. V. O.; Chaves, M. C. O.; da Cunha, E. V. L.; Gray, A. I.; Phytochemistry 1998, 49, 1381.

13. Ye, Chun L.; Yan-Hua, L.; Dong-Zhi, W.; Phytochemistry 2004, 65,445 .

14. Asai, T.; Hara, N.; Kobayashi, S.; Fujimoto, Y.; Phytochemistry 2008, 69, 1234.

15. Alavez-Solano, D.; Reyes-Chilpa, R.; Jiménez-Estrada, M.; Gómez-Garibay, F.; Chavez-Uribe, I.; Sousa-Sánchez, M.; Phytochemistry 2000, 55, 953.

16. Quintin, J.; Desrivot, J.; Thoret, S.; Le Menez, P.; Cresteil, T.; Lewin, G.; Bioorg. Med. Chem. Lett. 2009, 19, 167.

17. Gupta, S. R.; Seshadri, T. R.; Good, G. R.; Indian J. Chem. 1975, 13B, 632.

18. Gupta, B. K.; Gupta, G. K.; Dhar, K. L.; Atal, C. K.; Phytochemistry 1980, 19, 2034.

19. Miles, D. H.; de Medeiros, J. M. R.; Chittawong, V.; Hedin, P. A.; Swithenbank, C.; Lidert, Z.; Phytochemistry 1991, 30, 1131.

20. Calzada, F.; Mata, R.; Bye, R.; Linares, E.; Phytochemistry 1990, 29, 2737.

21. Silva, T. M. S.; Carvalho, M. G.; Braz-Filho, R.; Quim. Nova 2009, 32, 1119.

Submitted: December 8, 2009

Published online: April 19, 2011 


\section{Supplementary Information}

\section{Three New Compounds from Piper montealegreanum Yuncker (Piperaceae)}

Harley da S. Alves, Maria de F. V. de Souza and Maria C. de O. Chaves*

Laboratório de Tecnologia Farmacêutica, Universidade Federal da Paraíba, CP 5009, 58051-970 João Pessoa-PB, Brazil

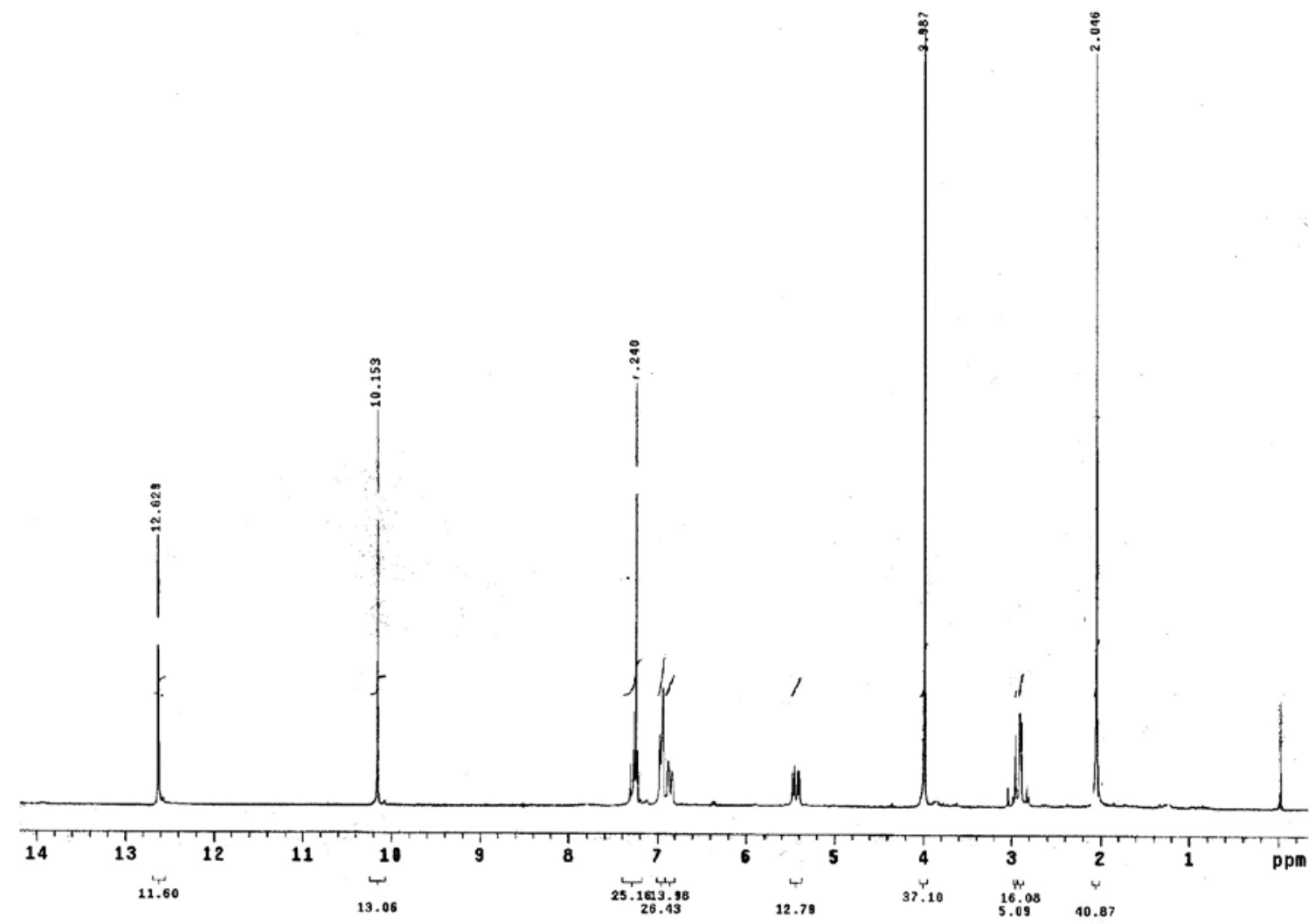

Figure S1. ${ }^{1} \mathrm{H}$ NMR spectrum $\left(\delta, \mathrm{CDCl}_{3}, 200 \mathrm{MHz}\right)$ of 1. 


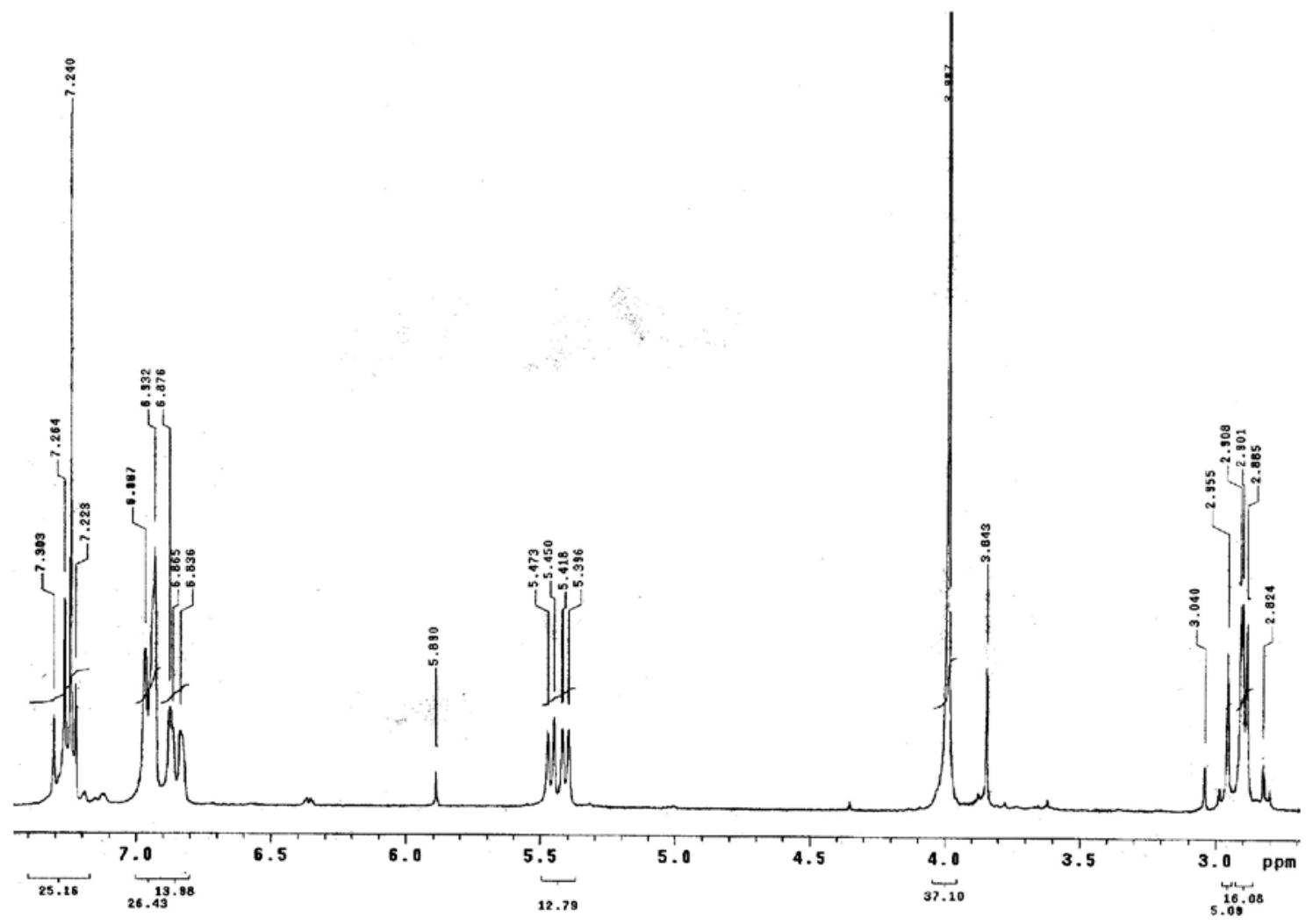

Figure S2. ${ }^{1} \mathrm{H}$ NMR spectrum $\left(\delta 8.0-2.8 \mathrm{ppm}, \mathrm{CDCl}_{3}, 200 \mathrm{MHz}\right)$ of $\mathbf{1}$.

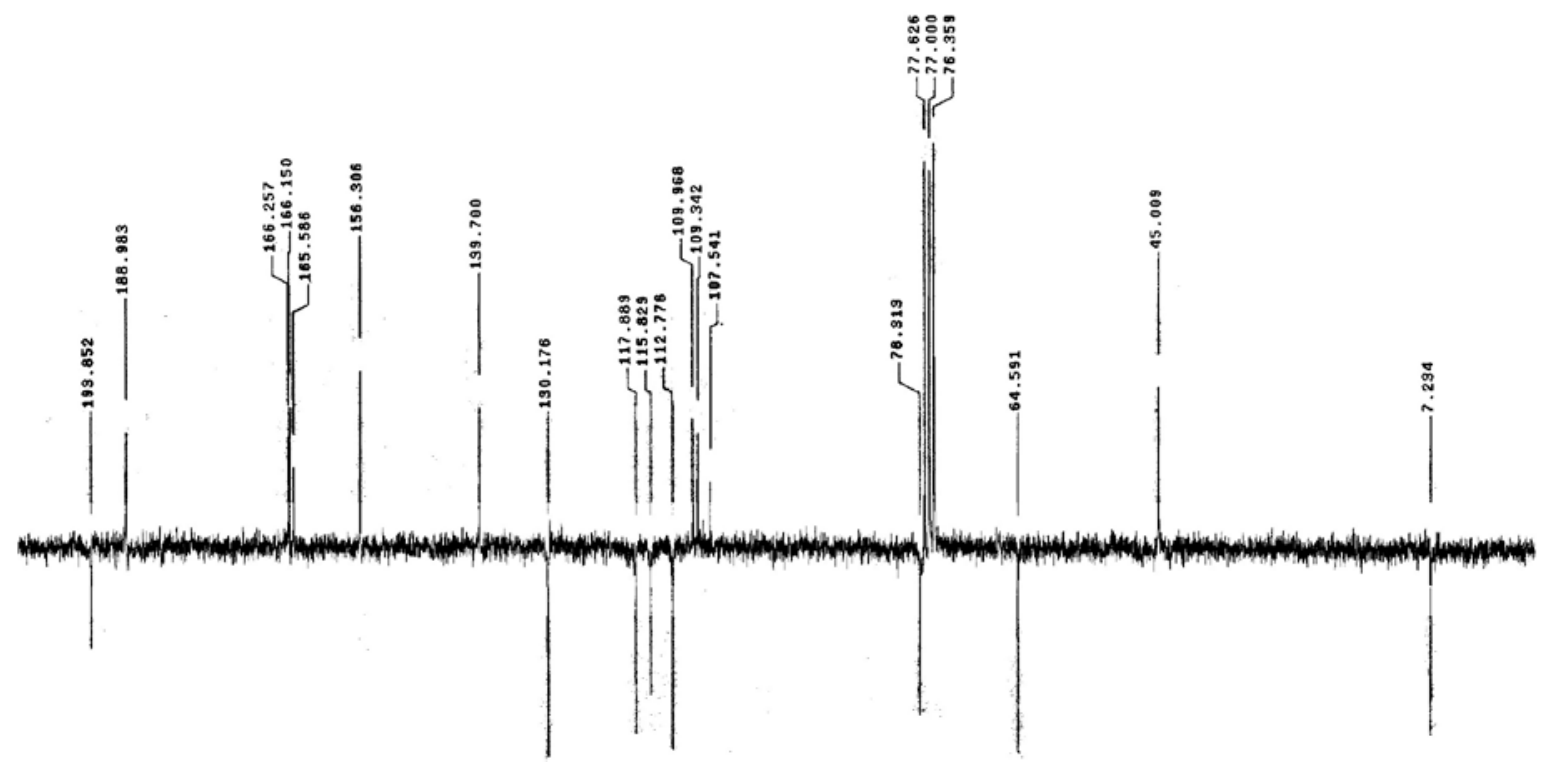

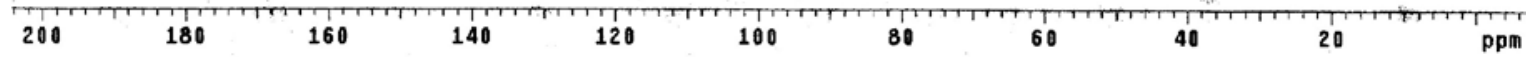

Figure S3. ${ }^{13} \mathrm{C}$ NMR (APT) spectrum $\left(\delta, \mathrm{CDCl}_{3} 50 \mathrm{MHz}\right)$ of $\mathbf{1}$. 


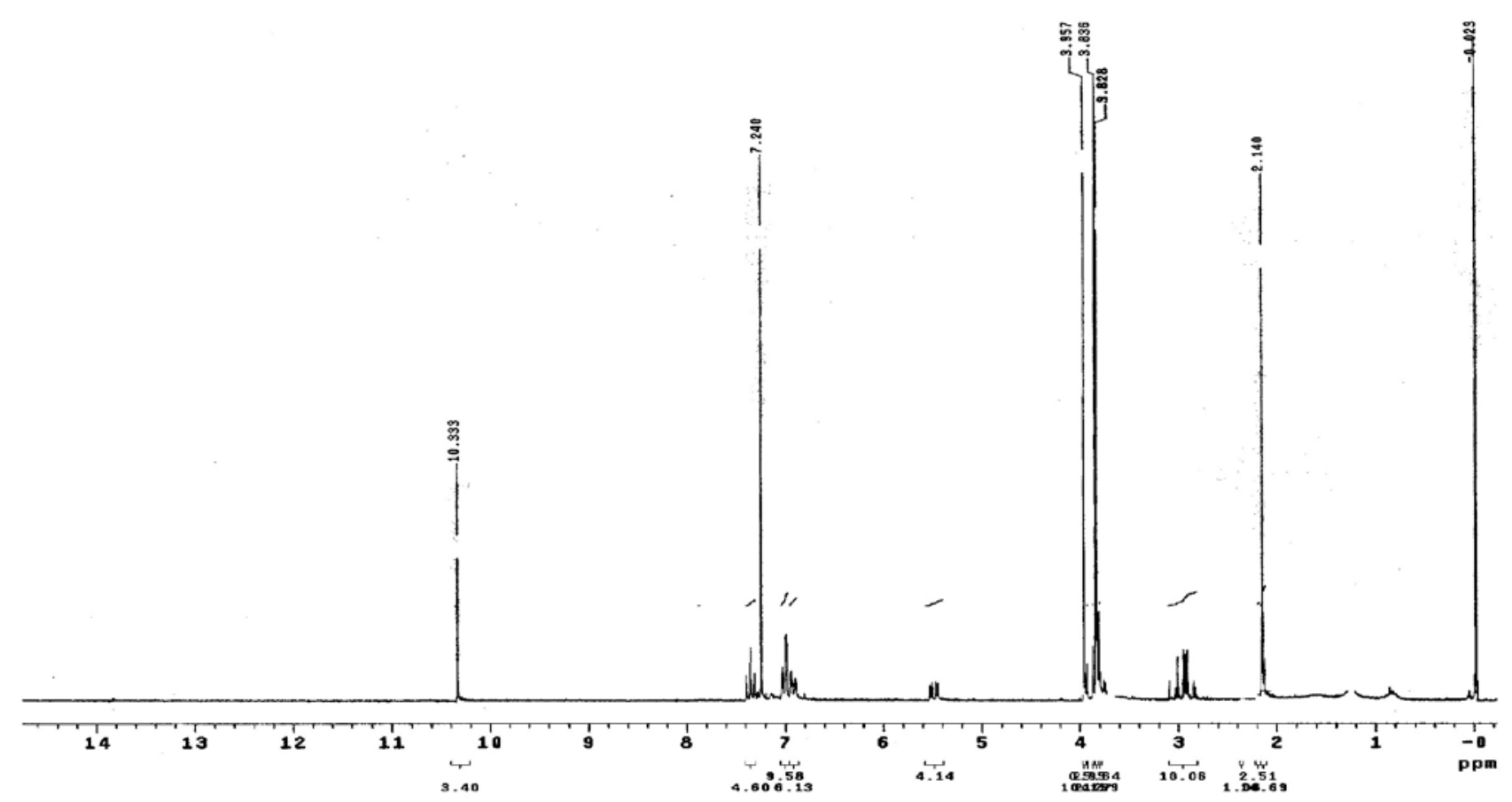

Figure S4. ${ }^{1} \mathrm{H}$ NMR spectrum $\left(\delta, \mathrm{CDCl}_{3}, 200 \mathrm{MHz}\right)$ of 1a.

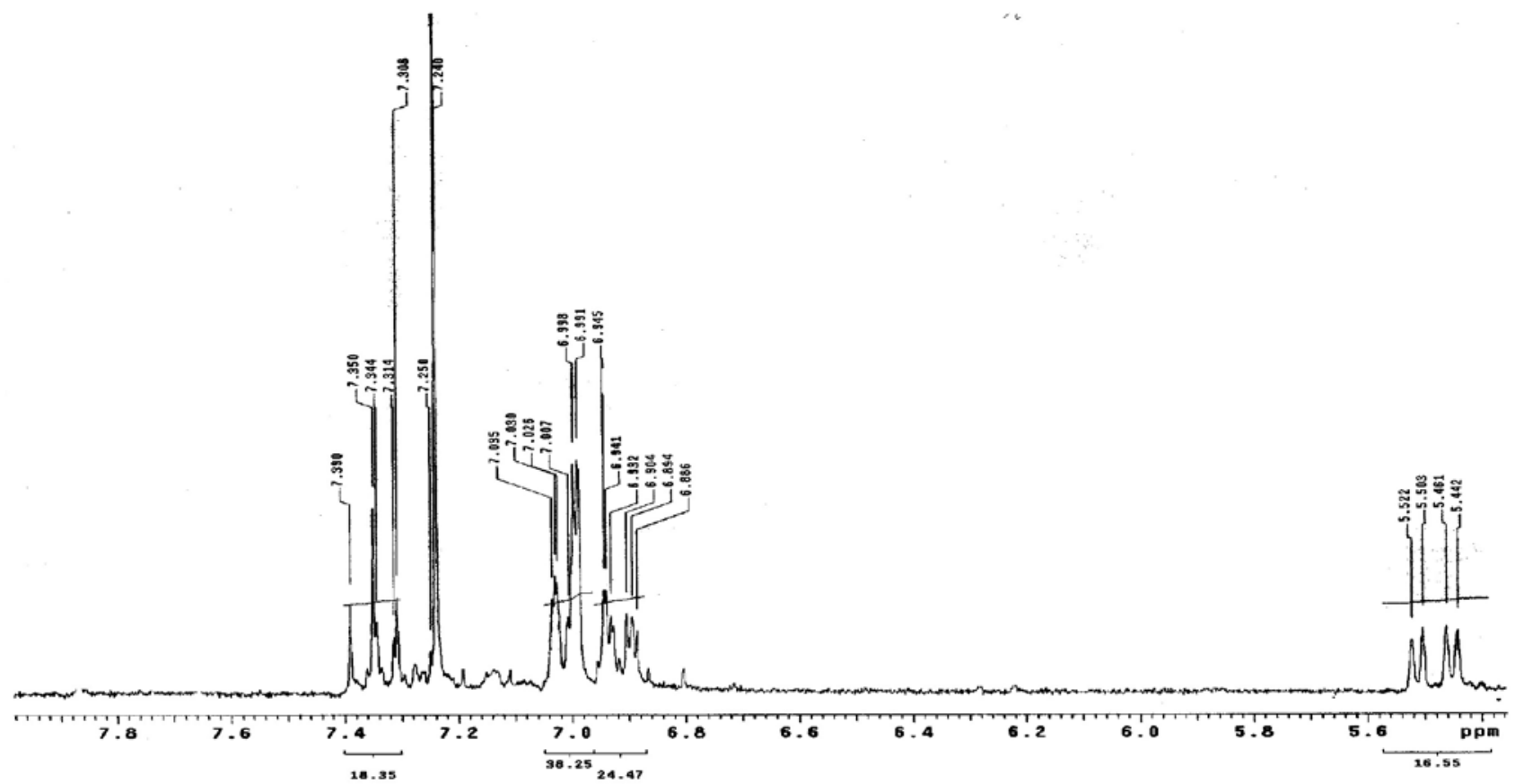

Figure S5. ${ }^{1} \mathrm{H}$ NMR spectrum $\left(\delta\right.$ 7.8-5.4 ppm, $\left.\mathrm{CDCl}_{3}, 200 \mathrm{MHz}\right)$ of $\mathbf{1 a}$. 

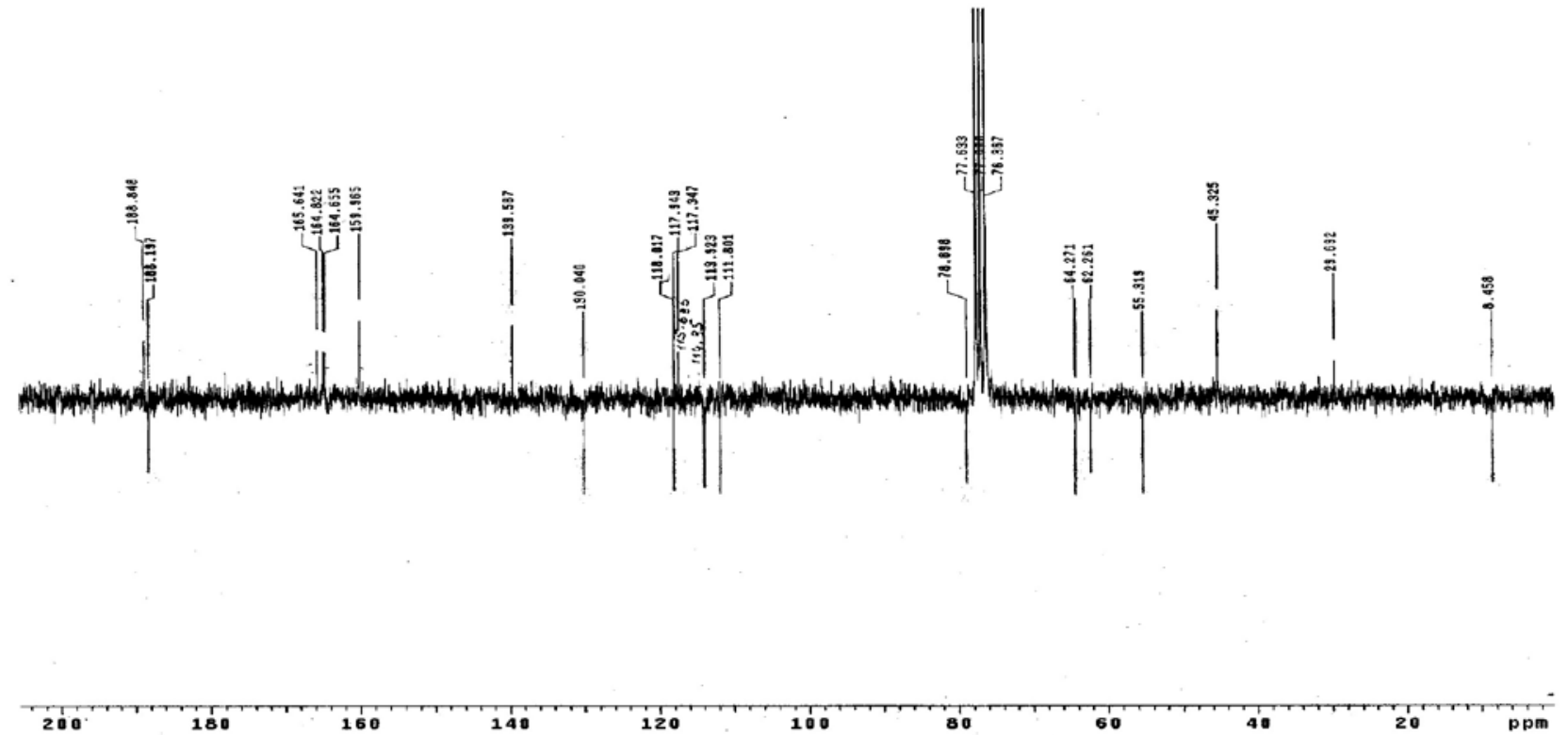

Figure S6. ${ }^{13} \mathrm{C}$ NMR (APT) spectrum $\left(\delta, \mathrm{CDCl}_{3} 50 \mathrm{MHz}\right)$ of $\mathbf{1 a}$

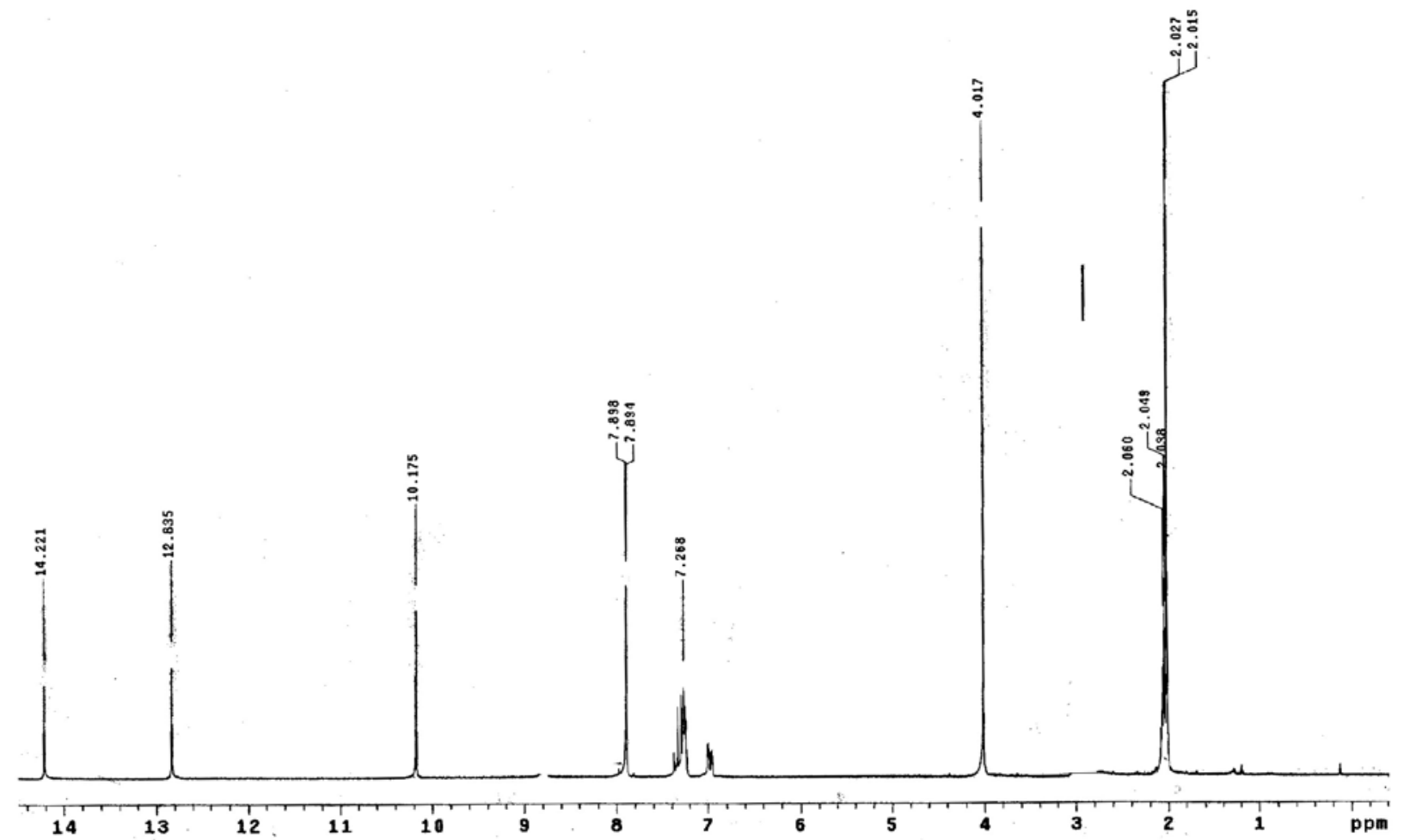

Figure S7. ${ }^{1} \mathrm{H}$ NMR spectrum $\left(\delta,\left(\mathrm{CD}_{3}\right)_{2} \mathrm{CO}, 200 \mathrm{MHz}\right)$ of 2. 


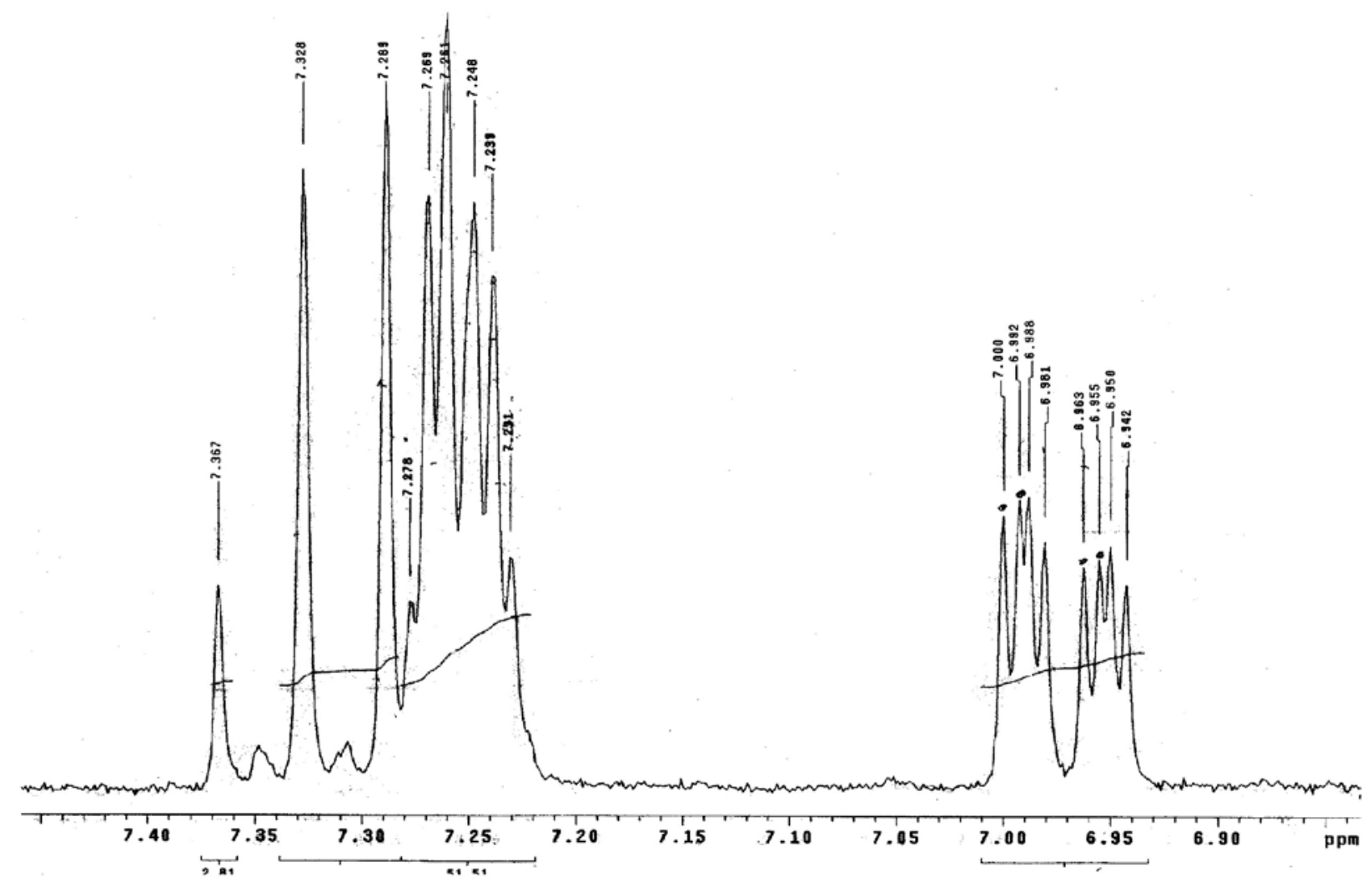

Figure S8. ${ }^{1} \mathrm{H}$ NMR spectrum $\left(\delta\right.$ 7.4-6.8 ppm, $\left.\left(\mathrm{CD}_{3}\right)_{2} \mathrm{CO}, 200 \mathrm{MHz}\right)$ of 2.
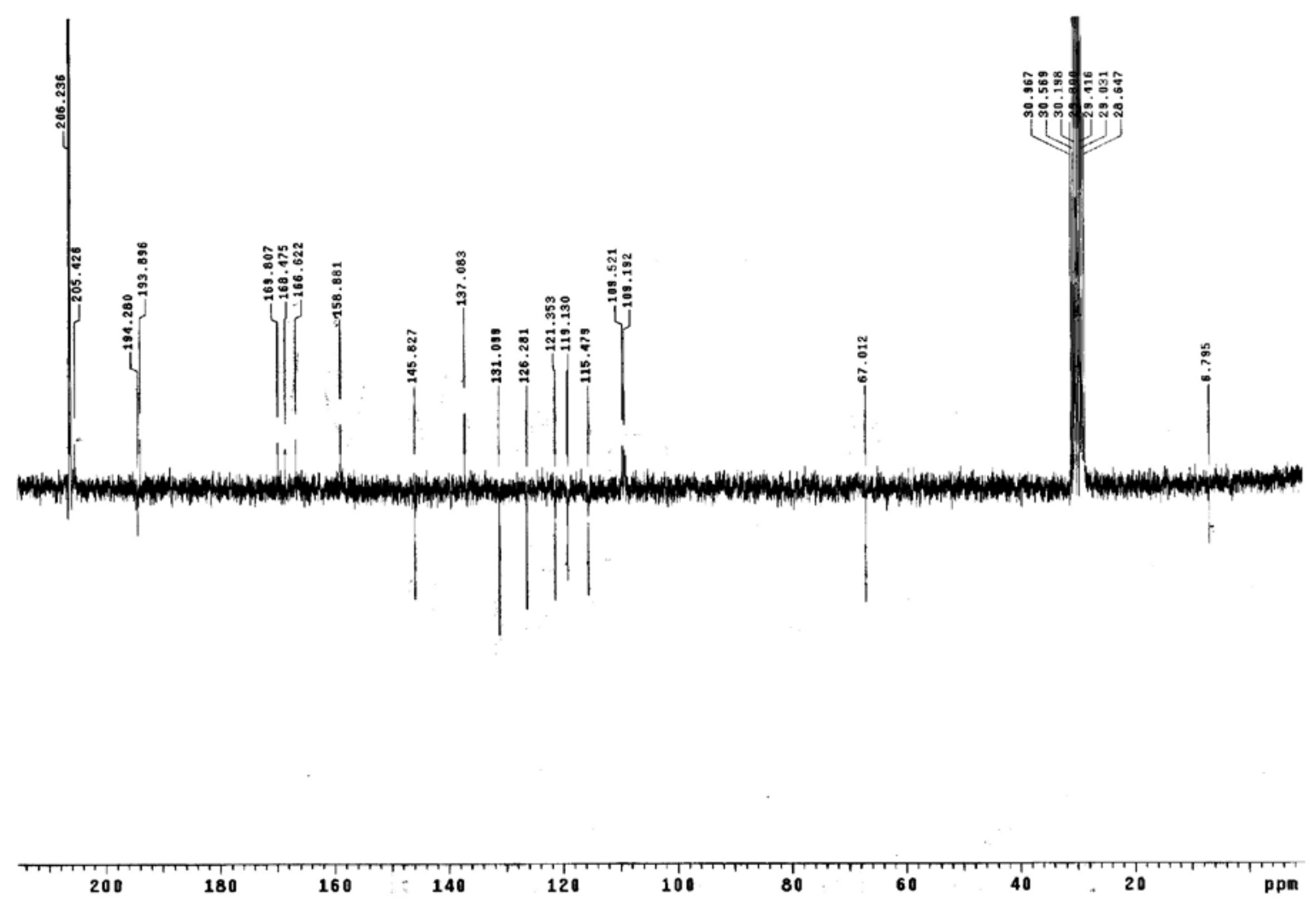

Figure S9. ${ }^{13} \mathrm{C}$ NMR (APT) spectrum $\left(\delta,\left(\mathrm{CD}_{3}\right)_{2} \mathrm{CO}, 50 \mathrm{MHz}\right)$ of 2. 


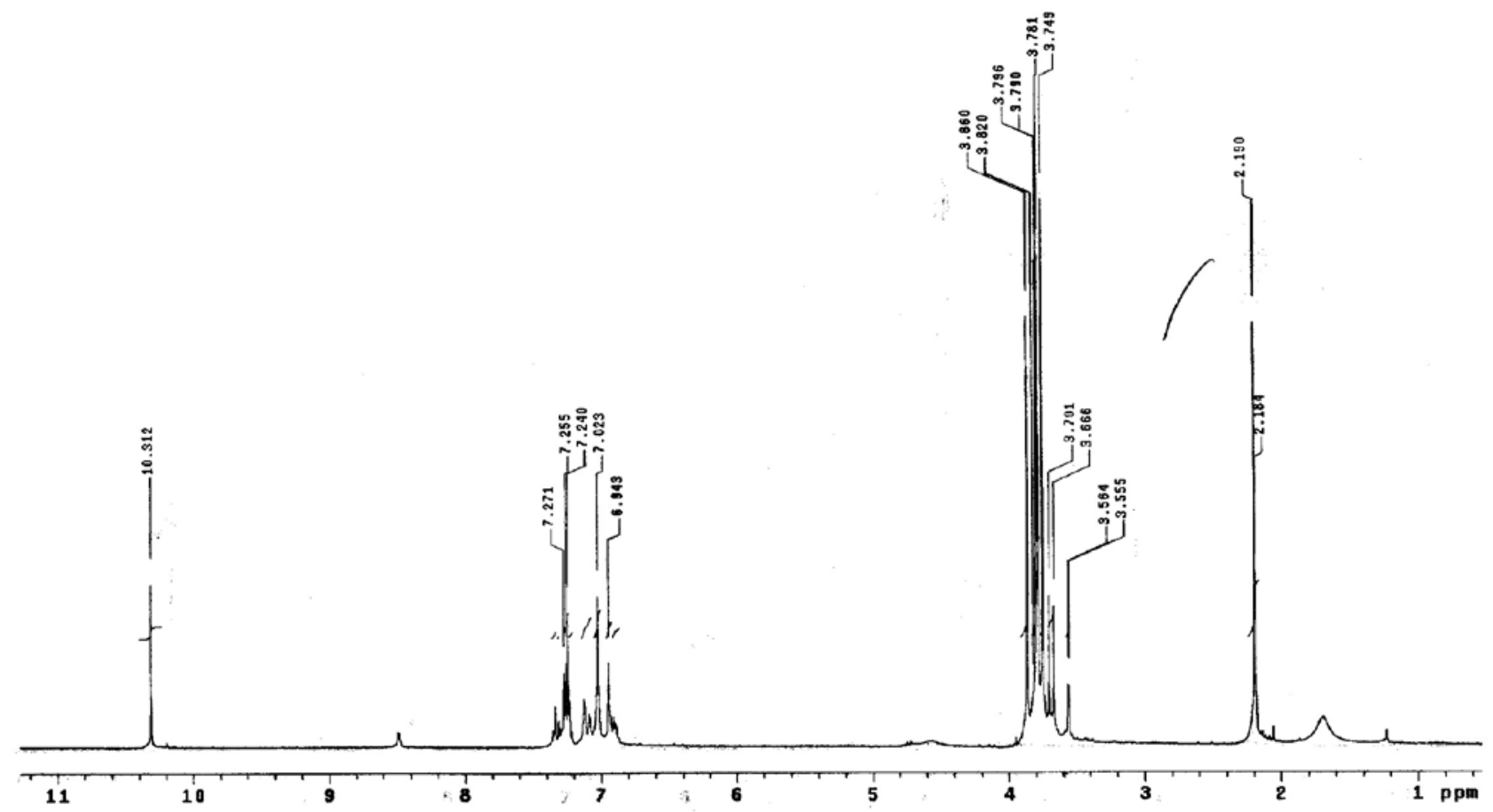

Figure S10. ${ }^{1} \mathrm{H}$ NMR spectrum $\left(\delta, \mathrm{CDCl}_{3}, 200 \mathrm{MHz}\right)$ of $\mathbf{2 a}$.

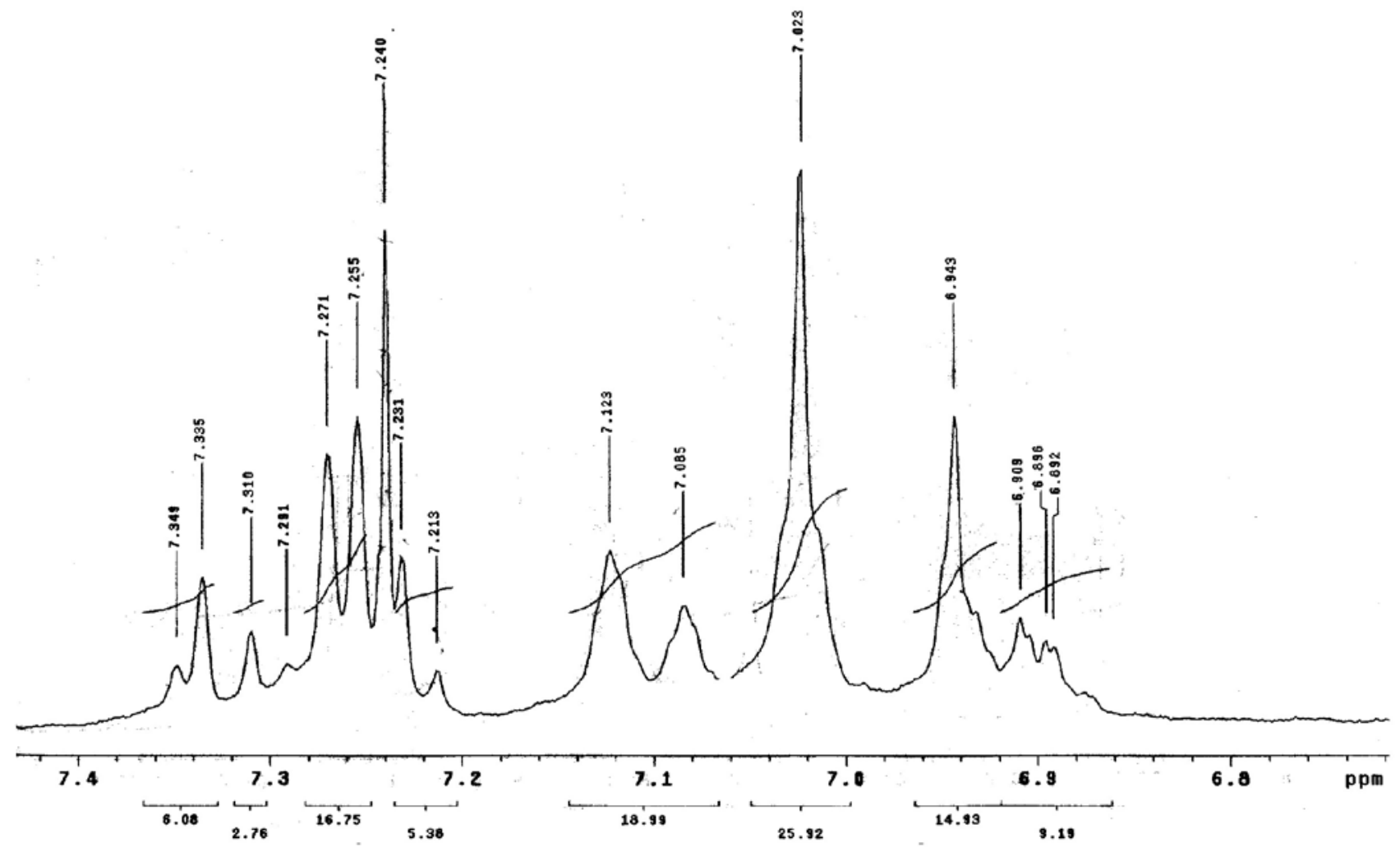

Figure S11. ${ }^{1} \mathrm{H}$ NMR spectrum $\left(\delta\right.$ 7.4-6.8 ppm, $\left.\mathrm{CDCl}_{3}, 200 \mathrm{MHz}\right)$ of $\mathbf{2 a}$. 


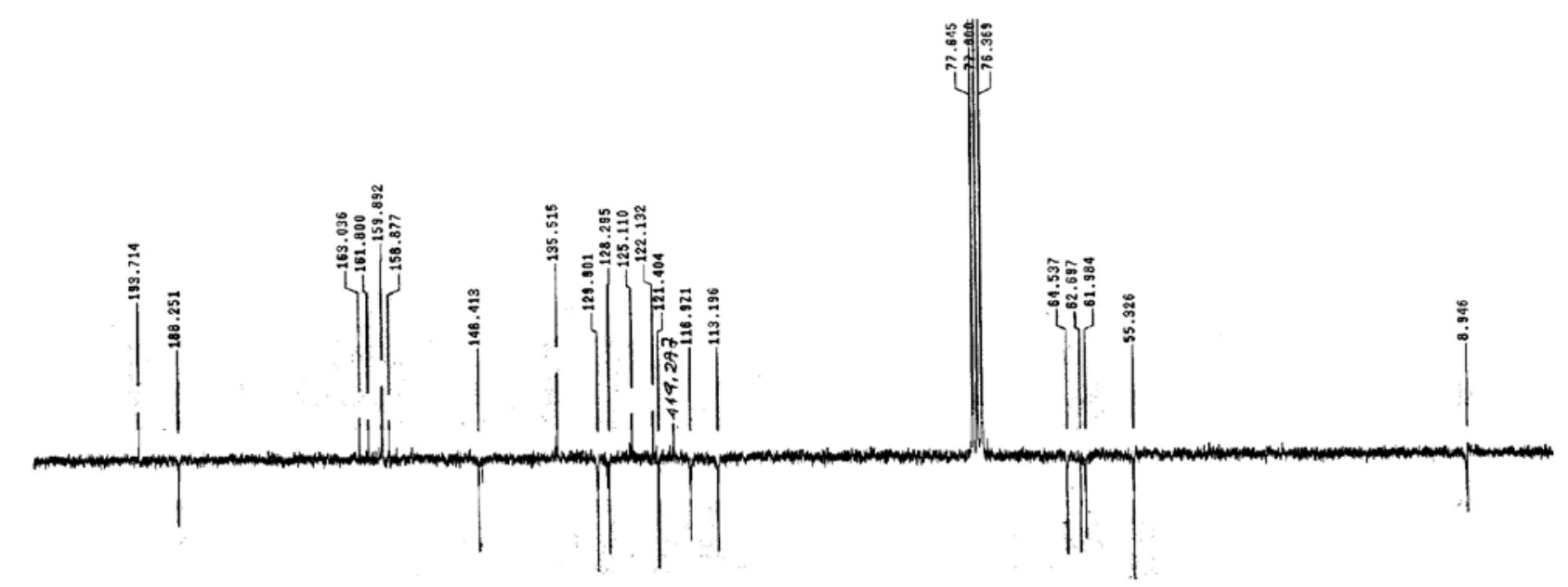

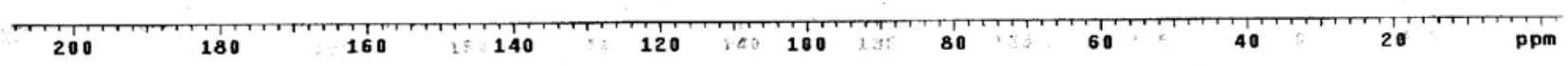

Figure S12. ${ }^{13} \mathrm{C}$ NMR (APT) spectrum $\left(\delta, \mathrm{CDCl}_{3} 50 \mathrm{MHz}\right)$ of $\mathbf{2 a}$.

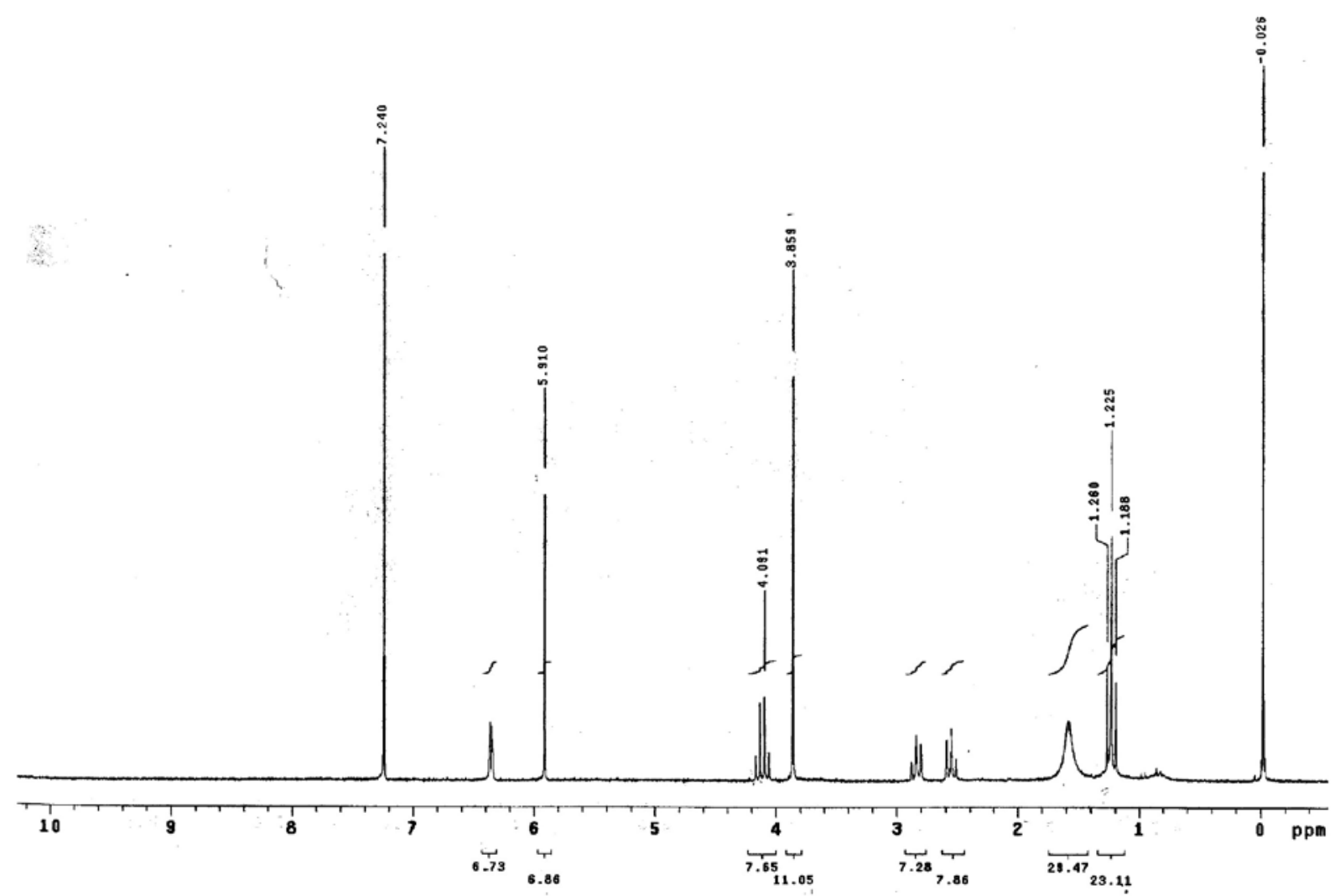

Figure S13. ${ }^{1} \mathrm{H}$ NMR spectrum $\left(\delta, \mathrm{CDCl}_{3}, 200 \mathrm{MHz}\right)$ of $\mathbf{3}$. 


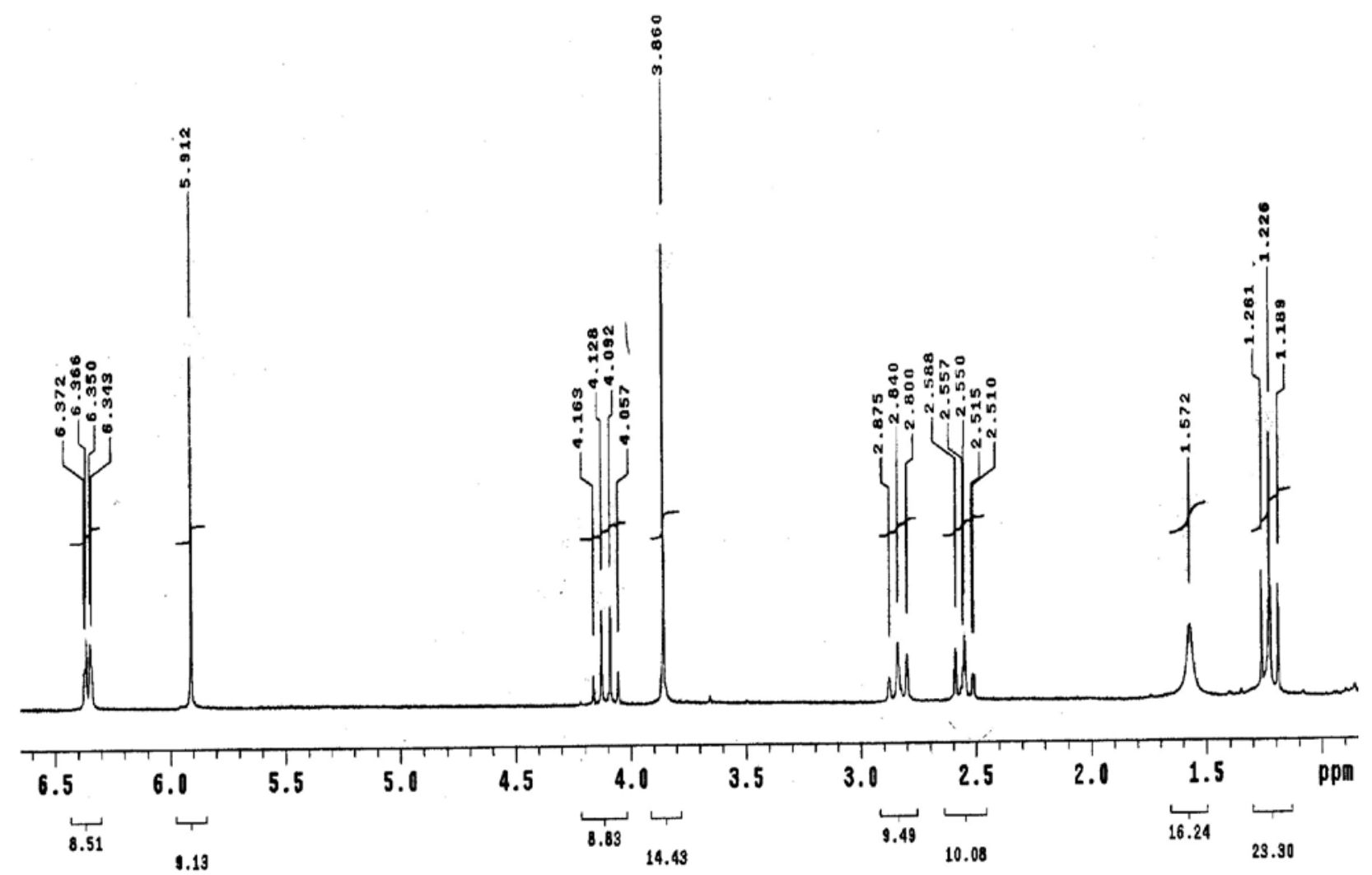

Figure S14. ${ }^{1} \mathrm{H}$ NMR spectrum $\left(\delta 6.5-1.0 \mathrm{ppm}, \mathrm{CDCl}_{3}, 200 \mathrm{MHz}\right)$ of $\mathbf{3}$.

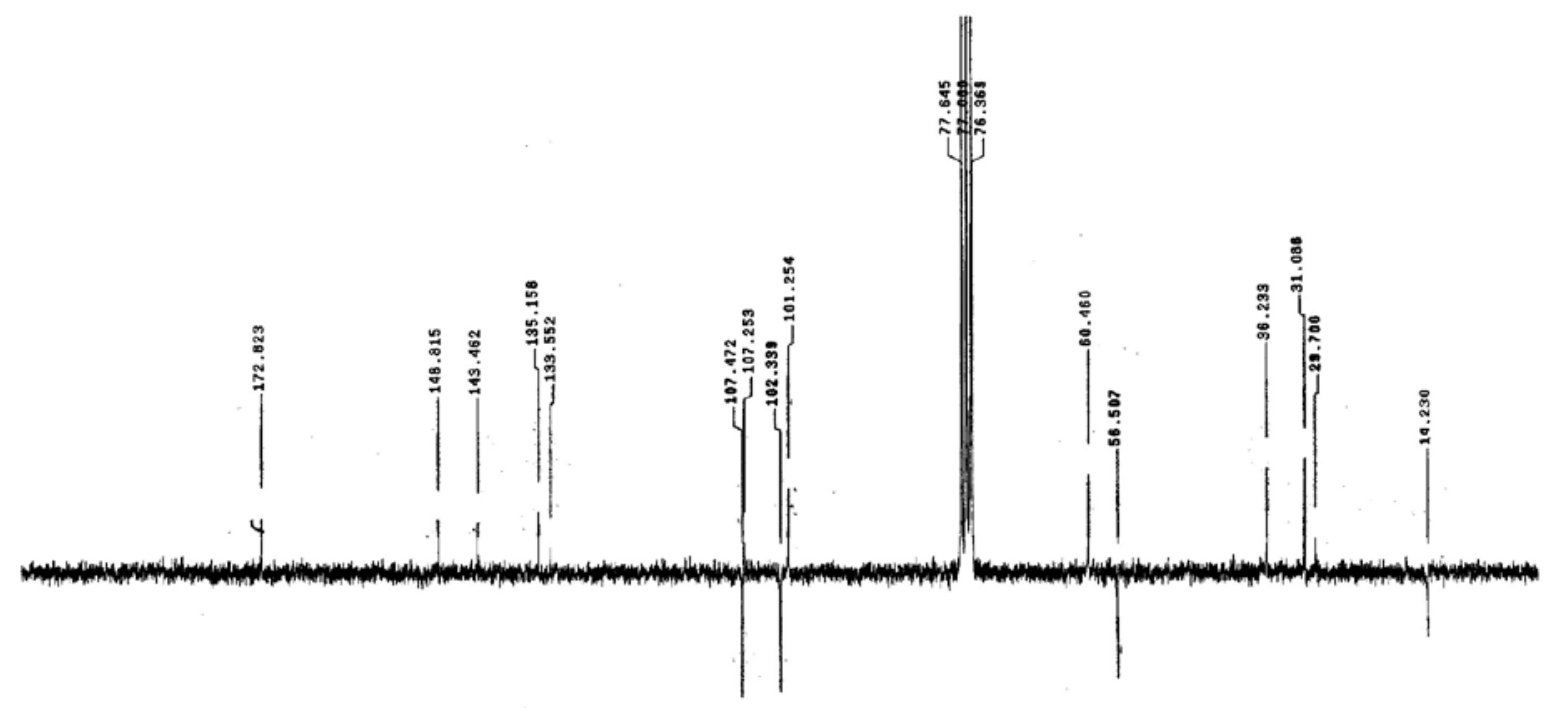

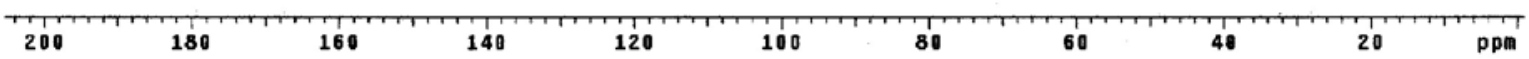

Figure S15. ${ }^{13} \mathrm{C}$ NMR (APT) spectrum $\left(\delta, \mathrm{CDCl}_{3}, 50 \mathrm{MHz}\right)$ of 3. 